\section{Jahre Fortschritte in der Arzneimitteltherapie: Mammakarzinom}

\author{
N. Harbeck ${ }^{1}$ \\ ${ }^{1}$ Leitung Brustzentrum der Klinik und Poliklinik für \\ Frauenheilkunde, Klinikum der Universität München (LMU)
}

Das Mammakarzinom ist bereits bei Erstdiagnose eine potenziell systemische Erkrankung, d.h. die medikamentöse Therapie ist ein essenzieller Bestandteil des multidisziplinären Therapiekonzeptes. An der Tatsache, dass das Mammakarzinom heute zu einer der heilbaren Tumorerkrankungen gehört, hat die Arzneimitteltherapie einem großen Anteil. In den westlichen Industrieländern sinkt seit Jahren die Sterblichkeit an dieser Erkrankung - als ausschlaggebend hierfür gilt neben dem Mammografiescreening die vorbeugende (adjuvante) Systemtherapie.

Als wichtige Säulen der Systemtherapie stehen beim Mammakarzinom Chemotherapie, Antihormontherapie und in den letzten Jahren vermehrt auch zielgerichtete Therapeutika zur Verfügung. Für die Chemotherapie können neben Taxanen und Anthrazyklinen, die als die wirksamsten Substanzgruppen bei Brustkrebs gelten, zahlreiche weitere wirksame Chemotherapeutika v.a. für die metastasierte Erkrankungssituation eingesetzt werden. Die Weiterentwicklungen auf diesem Gebiet betreffen v.a. den personalisierten Einsatz von Chemotherapien. Bei der frühen Erkrankungssituation bedeutet dies die Vermeidung von Über-, aber auch von Untertherapie durch den Einsatz von Multigensignaturen bei der Indikationsstellung sowie die Vermeidung von Spättoxizitäten. In der fortgeschrittenen Erkrankungssituation kann durch den Einsatz sequenzieller Monochemotherapien eine Symptomlinderung und Tumorregression erreicht werden. Auch als Kombinationspartner mit zielgerichteten Substanzen spielt die Chemotherapie nach wie vor eine wichtige Rolle.

Mit Hormonrezeptorstatus und HER2-Status und den gegen diese Zielstrukturen gerichteten Arzneimitteln bestimmt die Tumorbiologie heutzutage maßgeblich die systemtherapeutischen Konzepte.

Als Paradebeispiel der Weiterentwicklung der Systemtherapie bei Brustkrebs gilt die Einführung der anti-HER2-Therapien, durch die sich die Prognose bei diesem aggressiven Tumorsubtyp grundlegend verändert hat. Innerhalb von etwa 20 Jahren hat sich der Antikörper Trastuzumab nach den ersten präklinischen Experimenten als essenzieller Baustein der Mammakarzinomtherapie etabliert. Der monoklonale Antikörper Trastuzumab ist heute Standard in der metastasierten Situation, aber auch bei der vorbeugenden (adjuvanten) Therapie jeder HER2-positiven Mammakarzinomerkrankung im nicht metastasierten Stadium. Neue Daten zeigen, dass durch den kombinierten Einsatz der beiden anti-HER2Antikörper Trastuzumab und Pertuzumab in der metastasier- ten Situation das Überleben der Patientin weit über ein Jahr gegenüber dem bisherigen Standard verbessert werden kann. Aber auch in der präoperativen (neoadjuvanten) Therapie kommt es durch diese duale Blockade über z.B. 12 Wochen gemeinsam mit Chemotherapie zu pathologischen Komplettremissionen bei Operation von bis zu $90 \%$. Auch die Weiterentwicklung von Trastuzumab zum antibody-drug-conjugate T-DM1 führt zu Überlebensverbesserungen in der metastasierten Situation, auch in späten Therapielinien. Durch die gute Verträglichkeit dieser Substanz ist eine Ausweitung der Therapieindikationen für T-DM1 auch auf die frühe Erkrankungssituation durch klinische Studien wünschenswert.

Die Antihormontherapie ist die älteste zielgerichtete Therapie beim Mammakarzinom. Nach den ersten operativen Beispielen Ende des 20. Jahrhunderts gibt es heute mit Tamoxifen, Aromatasehemmstoffen oder Fulvestrant mehrere Therapieoptionen, die in der Regel sequenziell eingesetzt werden. Seit Anfang dieser Dekade gibt es mit den ersten Daten zur mTOR Inhibition (Everolimus) in Kombination mit einem Aromatasehemmer den Nachweis der Überwindung endokriner Resistenz durch die Kombination mit einer zielgerichteten Therapie. Dies ermöglicht die längere antihormonell basierte Therapie und das Herausschieben von Chemotherapie in der metastasierten Situation bei Hormonrezeptor-positiver HER2-negativer Erkrankung. Die neuesten Daten zur CDK4/6Inhibition zeigen, dass diese Substanzgruppe auch die Zeit bis zur Progression in der metastasierten Situation nahezu verdoppeln kann. Neben der ersten Zulassung für Palbociclib gibt es hier zwei weitere Substanzen (Ribociclib, Abemaciclib), für die Zulassungsstudien bereits abgeschlossen sind. Weitere zielgerichtete Substanzen sind in dieser Therapieindikation gemeinsam mit Antihormontherapie in der Entwicklung.

Durch die Fortschritte in der Medikamentenentwicklung hat sich die Prognose beim Mammakarzinom in den letzten 50 Jahren dramatisch verbessert. Die Heilungschancen bei Ersterkrankung betragen heute zwischen $70-80 \%$, und auch die Überlebenschancen in der metastasierten Situation sind bei einigen Subtypen bereits deutlich besser geworden. Bei der frühen Erkrankung geht die Forschung in Richtung personalisierte Therapiekonzepte in Abhängigkeit von der Tumorbiologie und der Beurteilung eines frühen Ansprechens in der präoperativen Therapiesituation. In der metastasierten Erkrankung besteht durch die neuen Entwicklungen für viele Patientinnen die berechtigte Hoffnung auf eine lang andauernde Therapiemöglichkeit durch zielgerichtete Substanzen im Sinne einer chronischen Erkrankung. Neue Studienkonzepte konzentrieren sich hier auch auf die Entwicklung von Biomarkern gemeinsam mit neuen Medikamenten, um eine gezieltere Therapiewahl zu ermöglichen. Wichtig für den weiteren Fortschritt ist die konsequente und rasche Weiterentwicklung der Therapieoptionen in kontrollierten klinischen Studien in Kooperation zwischen Industrie und akademischen Studiengruppen.

Interessenkonflikte: Honorare für Beratung und Vorträge von: Amgen, AstraZeneca, Celgene, Novartis, Pfizer, Roche und Sandoz. 


\section{Bibliografie}

DOI http://dx.doi.org/10.1055/s-0042-112405

Drug Res 2016; 66, Suppl. 1: S29-S30

(c) Georg Thieme Verlag KG Stuttgart · New York . ISSN 2194-9379
Korrespondenzadresse

Prof. Dr. med. Nadia Harbeck

Leitung Brustzentrum der Klinik und

Poliklinik für Frauenheilkunde

Klinikum der Universität München (LMU)

Marchioninistraße 15

81377 München

nadia.harbeck@med.uni-muenchen.de

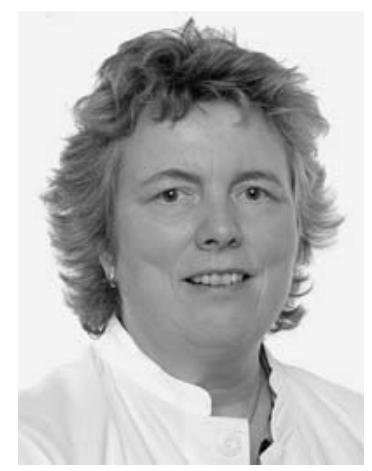

